Silva Filho, PSP, Costa, REAR, Santos, MBL, Lima, SPN, Silva, LA, Freitas, AS, Silva, JP, Araújo, LKC, Cunha, SH, Araújo, FWC, Silva, APA, Alves, DRCF, Mendes, AM, Leão, CA, Kirchesch, CL \& Macedo, JL, (2020). Management of patients with cancer during the COVID19 pandemic. Research, Society and Development, 9(7): 1-17, e628974609.

\title{
Gerenciamento dos pacientes com câncer durante a pandemia de COVID-19
}

Management of patients with cancer during the COVID-19 pandemic

Manejo de pacientes con cáncer durante la pandemia de COVID-19

Recebido: 16/05/2020 | Revisado: 19/05/2020 | Aceito: 21/05/2020 | Publicado: 29/05/2020

Paulo Sérgio da Paz Silva Filho

ORCID: https://orcid.org/0000-0003-4104-6550

Centro Universitário Maurício de Nassau, Brasil

E-mail: pauloosergio1@ outlook.com

Rafael Everton Assunção Ribeiro da Costa

ORCID: https://orcid.org/0000-0002-0798-890X

Universidade Estadual do Piauí, Brasil

E-mail: rafassuncao.rafael@gmail.com

Monyka Brito Lima dos Santos

ORCID: https://orcid.org/0000-0002-6866-9435

Centro Universitário de Ciências e Tecnologia do Maranhão, Brasil

E-mail: monyka.brito@hotmail.com

Sonia Pantoja Nascimento Lima

ORCID: https://orcid.org/0000-0003-0657-0031

Centro Universitário de Ciencias e Tecnologia do Maranhão, Brasil

E-mail: soniapantoja_s@hotmail.com

Leticia de Almeida da Silva

ORCID: https://orcid.org/0000-0003-4435-6909

Universidade Federal do Piauí, Brasil

E-mail: leticia.micheli14@hotmail.com

Ananda Santos Freitas

ORCID: https://orcid.org/0000-0002-6420-3945

Universidade Estadual do Maranhão, Brasil

E-mail: annandhacx@hotmail.com 
Joélio Pereira da Silva

ORCID: https://orcid.org/0000-0002-8182-2000

Universidade Estadual do Ceará, Brasil

E-mail: joeliops@hotmail.com

\section{Laianny Karola Carvalho de Araújo}

ORCID: https://orcid.org/0000-0003-3033-3255

Universidade Federal do Piauí, Brasil

E-mail: laianny.k.carvalho@hotmail.com

Simone Helena Cunha

ORCID: https://orcid.org/0000-0002-7318-4678

Universidade Federal do Piauí, Brasil

E-mail: simonehelena@gmail.com

Francy Waltilia Cruz Araújo

ORCID: https://orcid.org/0000-0001-5961-2528

Universidade Federal do Piauí, Brasil

E-mail: francy_cruz@hotmail.com.br

Ana Paula Alves da Silva

ORCID: https://orcid.org/0000-0003-0946-447X

Universidade Estadual do Maranhão, Brasil E-mail: lylinha16@hotmail.com

Darci Rosane Costa Freitas Alves

ORCID: https://orcid.org/0000-0001-7839-9996

Universidade Estadual do Maranhão, Brasil

E-mail:darci_rane@hotmail.com

Annarelly Morais Mendes

ORCID: https://orcid.org/0000-0002-4367-8888

Universidade Estadual do Maranhão, Brasil

E-mail: annarellymorais1@gmail.com

Chiara de Aquino Leão

ORCID: https://orcid.org/0000-0001-8669-9008

Centro Universitário UniFacid Wyden, Brasil

E-mail: chiaraleao@icloud.com 


\section{Cryshna Leticia Kirchesch}

ORCID: https://orcid.org/0000-0002-5866-3216

Universidade Federal de Pelotas, Brasil

E-mail: cryshna.kirchesch@ebserh.gov.br

Juliana Leal Macedo

ORCID: https://orcid.org/0000-0002-6593-9073

Instituto de Ensino Superior do Vale do Parnaíba, Brasil

E-mail: julianalpinheiromachado@gmail.com

\section{Resumo}

A presente pesquisa teve como objetivo descrever o gerenciamento de pacientes com câncer durante a pandemia de COVID-19. O presente estudo se trata de uma pesquisa exploratória do tipo revisão de literatura. A realização das buscas ocorreu entre fevereiro a abril de 2020, utilizando as bases de dados Science Direct, Lilacs e MEDLINE, com o recorte temporal de 2014 a 2020. Houve uma seleção criteriosa no que diz respeito às obras utilizadas para o desenvolvimento desta revisão. Os descritores utilizados, de modo associado e isolado, foram: Oncologia, coronavírus, cuidados, prevenção e COVID-19, em inglês, espanhol e português e indexados no DeCS. Os critérios de exclusão foram: trabalhos científicos com apenas resumos disponíveis, publicações duplicadas, outras metodologias, como artigos reflexivos, editoriais, comentários, cartas ao editor, artigos incompletos, e aqueles que não se enquadravam dentro da proposta oferecida para o tema e/ou estavam fora do recorte temporal. Houve utilização de teses e dissertações. Dentro das buscas, foram encontrados 520 artigos; porém, após a exclusão de achados duplicados e incompletos, restringiu-se a 62 obras, estas foram lidas individualmente pelos pesquisadores. Ao final das análises, 11 artigos foram utilizados na revisão. Conclui-se que os pacientes com tumores apresentam maior risco de COVID-19 que pacientes nãotumorais. Os sintomas restantes são semelhantes aos pacientes não-tumorais: mialgia e fadiga. Contudo, as manifestações de imagem são mais graves e as complicações mais sérias são mais prováveis. Quanto maior a idade, maior o risco de eventos clínicos graves e pior o prognóstico. Palavras-chave: Oncologia; Coronavírus; Cuidados; Prevenção; COVID-19.

\footnotetext{
Abstract

This research aimed to describe the management of cancer patients during the COVID-19 pandemic. The present study is an exploratory research of the literature review type. The
} 
searches were carried out between February and April 2020, using the databases Science Direct, Lilacs and MEDLINE, with the time frame from 2014 to 2020. There was a careful selection with respect to the works used for the development of this review. The descriptors used, in an associated and isolated way, were: Oncology, coronavirus, care, prevention and COVID-19, in English, Spanish and Portuguese and indexed in DeCS. The exclusion criteria were: scientific papers with only available abstracts, duplicate publications, other methodologies, such as reflective articles, editorials, comments, letters to the editor, incomplete articles, and those that did not fit within the proposal offered for the theme and / or were outside the time frame. Theses and dissertations were used. Within the searches, 520 articles were found; however, after excluding duplicate and incomplete findings, it was restricted to 62 works, these were read individually by the researchers. At the end of the analyzes, 11 articles were used in the review. It is concluded that patients with tumors have a higher risk of COVID-19 than non-tumor patients. The remaining symptoms are similar to non-tumor patients: myalgia and fatigue. However, image manifestations are more serious and more serious complications are more likely. The older you are, the greater the risk of serious clinical events and the worse the prognosis.

Keywords: Oncology; Coronavirus; Care; Prevention; COVID-19.

\section{Resumen}

Esta investigación tuvo como objetivo describir el manejo de pacientes con cáncer durante la pandemia de COVID-19. El presente estudio es una investigación exploratoria del tipo de revisión de literatura. Las búsquedas se llevaron a cabo entre febrero y abril de 2020, utilizando las bases de datos Science Direct, Lilacs y MEDLINE, con el marco temporal de 2014 a 2020. Hubo una selección cuidadosa con respecto a los trabajos utilizados para el desarrollo de este revisión. Los descriptores utilizados, de forma asociada y aislada, fueron: oncología, coronavirus, cuidados, prevención y COVID-19, en inglés, español y portugués e indexados en DeCS. Los criterios de exclusión fueron: artículos científicos con solo resúmenes disponibles, publicaciones duplicadas, otras metodologías, como artículos reflexivos, editoriales, comentarios, cartas al editor, artículos incompletos y aquellos que no encajaban en la propuesta ofrecida para el tema y / o estaban fuera del marco de tiempo. Se utilizaron tesis y disertaciones. Dentro de las búsquedas, se encontraron 520 artículos; sin embargo, después de excluir hallazgos duplicados e incompletos, se restringió a 62 trabajos, los investigadores los leyeron individualmente. Al final de los análisis, se utilizaron 11 artículos en la revisión. Se concluye que los pacientes con tumores tienen un mayor riesgo de COVID-19 que los pacientes no 
tumorales. Los síntomas restantes son similares a los pacientes no tumorales: mialgia y fatiga. Sin embargo, las manifestaciones de la imagen son más graves y las complicaciones más graves son más probables. Cuanto mayor sea, mayor es el riesgo de eventos clínicos graves y peor es el pronóstico.

Palabras clave: Oncología; Coronavirus; Cuidados, Prevención; COVID-19.

\section{Introdução}

Nos últimos anos, o coronavírus da Síndrome Respiratória Aguda Grave (SARS-CoV) e o coronavírus da Síndrome Respiratória do Oriente Médio (MERS-CoV) foram transmitidos de animais para humanos, ocasionando doenças respiratórias graves SARS e MERS em áreas endêmicas. Em dezembro de 2019, um novo coronavírus foi diagnosticado na cidade de Wuhan, na China, com capacidade de transmissão humano-humano. A doença denominada COVID-19 se espalhou rapidamente por todo o mundo, ocasionando uma pandemia (Hirano \& Murakami, 2020).

Os principais sintomas apresentados por pacientes diagnosticados com COVID-19 são: febre, tosse seca, fadiga, congestão nasal, dor de garganta e diarreia. A gravidade, na maioria das vezes, está relacionada à idade, sendo os idosos o principal grupo de risco, com ênfase em pessoas acima de 80 anos que tiveram uma taxa de mortalidade de casos (CFR) de $14,8 \%$. O CFR foi elevado em pacientes com comorbidades, incluindo doenças cardiovasculares, diabetes, doenças respiratórias crônicas, hipertensão e câncer (Fisher \& Heymann, 2020).

Geralmente, os pacientes diagnosticados com câncer estão sob imunossupressão por conta da invasão tumoral e do impacto da quimioterapia, cirurgia e outras terapêuticas empregadas, tornando-os mais suscetíveis à infecção por SARS-CoV-2. Porém, existe poucos estudos a respeito da continuidade da terapia antitumoral e da janela de tempo de tratamento após a infecção por SARS-CoV-2 em pacientes com tumor. A incidência de eventos adversos graves pós-clínicos é significativamente maior nos pacientes que não foram submetidos a algum tipo de tratamento. Esses relatos acarretam no atraso da terapia antitumoral e fortalecem a vigilância durante a infecção por SARS-CoV-2 (Ye et al., 2019).

O SARS-CoV-2 é transmitido, normalmente, por gotículas ou contato próximo, o que é agravado em aglomerações. Pacientes com tumor apresentam elevado risco para este patógeno por conta da sua função imunológica comprometida. A identificação precoce de pacientes com tumor e COVID-19 e a compreensão das suas características de distribuição ajudam a melhorar 
a taxa de cura e a controlar melhor a pandemia e o alastramento de SARS-CoV-2 (Zhaoet al., 2020).

Assim, a presente pesquisa teve como objetivo descrever o gerenciamento de pacientes com câncer durante a pandemia de COVID-19.

\section{Metodologia}

O presente estudo se trata de uma pesquisa exploratória do tipo revisão de literatura. Gil (2014, p. 50) indica que a pesquisa bibliográfica é: “aquela desenvolvida a partir de material já elaborado, constituído principalmente de livros e de artigos científicos, tendo como principal vantagem a cobertura de uma maior gama de fenômenos, se comparado a uma pesquisa direta circunscrita a um campo empírico”. Pereira, Shitsuka, Pereira, (2018) expõem que, na pesquisa bibliográfica, há a busca, leitura e análise dos textos com a discussão dos resultados obtidos a partir das referências utilizadas na elaboração do trabalho acadêmico ou científico.

Para o desenvolvimento desta revisão, foram percorridas as seguintes etapas: 1) definiçãao do tema e formulação da questão norteadora, 2) estabelecimento dos critérios de inclusão e exclusão, 3) definição dos descritores, 4) pré-seleção dos artigos, 5) avaliação dos estudos (interpretação dos resultados) e 6) apresentação da revisão.

As perguntas norteadoras da investigação utilizadas foram: "Quais os riscos de pacientes diagnosticados com câncer serem acometidos pela COVID-19?", "Quais os principais pacientes oncológicos durante a pandemia de COVID-19?" e "Qual o papel do profissional de saúde que atua no setor de Oncologia durante a pandemia?".

A realização das buscas ocorreu entre fevereiro a abril de 2020, utilizando as bases de dados Science Direct, Lilacs e MEDLINE, com o recorte temporal de 2014 a 2020. Houve uma seleção criteriosa no que diz respeito às obras utilizadas para o desenvolvimento desta revisão. Os descritores utilizados, de modo associado e isolado, foram: "oncologia", "coronavírus", “cuidados", "prevenção" e "COVID-19", em inglês, espanhol e português e indexados no DeCS (Descritores em Ciências da Saúde).

Os critérios de exclusão foram: trabalhos científicos com apenas resumos disponíveis, publicações duplicadas, outras metodologias, como artigos reflexivos, editoriais, comentários, cartas ao editor, artigos incompletos, e aqueles que não se enquadravam dentro da proposta oferecida para o tema e/ou estavam fora do recorte temporal. Houve utilização de teses e dissertações. 


\section{Resultados e Discussão}

Dentro das buscas, foram encontrados 520 artigos; porém, após a exclusão de achados duplicados e incompletos, restringiu-se a 62 obras, estas foram lidas individualmente pelos pesquisadores. Ao final das análises, 11 artigos foram utilizados na revisão, os quais possuíam os descritores inclusos no tema e/ou resumo e foram incluídos porque melhor se enquadravam no objetivo proposto.

O Quadro 1 demonstra todos os artigos finais selecionados para o estudo, com base no seu título, autores e seu ano de publicação, plataformas de busca e nas revistas na qual se encontram indexados. 
Quadro 1: Artigos selecionados para discussão da pesquisa (autor e ano, nome do artigo, objetivo e conclusão).

\begin{tabular}{|c|c|c|c|c|}
\hline $\mathbf{N}^{\circ}$ & NOME DO ARTIGO & AUTOR/ANO & PLATAFORMA & REVISTA \\
\hline 1 & $\begin{array}{l}\text { Infecções respiratórias } \\
\text { virais agudas em pacientes } \\
\text { pediátricos com câncer em } \\
\text { tratamento quimioterápico. }\end{array}$ & $\begin{array}{l}\text { Benites, E. C., Cabrini, D. } \\
\text { P., Silva, A. C., Silva, J. } \\
\text { C., Catalan, D. T., Berezin, } \\
\text { E. N., ... \& Passos, S. D. } \\
\text { (2014). }\end{array}$ & LILACS & Jornal de Pediatria \\
\hline 2 & $\begin{array}{l}\text { Resultados terapêuticos da } \\
\text { homeopatia em pacientes } \\
\text { suspeitos ou confirmados } \\
\text { de COVID-19 no Brasil: } \\
\text { protocolo para estudo } \\
\text { observacional prospectivo. }\end{array}$ & Dantas, F. (2020). & LILACS & BVS-Homeopatia \\
\hline 3 & $\begin{array}{l}\text { Q\&A: The novel } \\
\text { coronavirus outbreak } \\
\text { causing COVID-19. }\end{array}$ & $\begin{array}{l}\text { Fisher, D., \& Heymann, D. } \\
\qquad(2020) .\end{array}$ & MEDLINE & BMC medicine \\
\hline 4 & $\begin{array}{c}\text { COVID-19: A New Virus, } \\
\text { but a Familiar Receptor } \\
\text { and Cytokine Release } \\
\text { Syndrome. }\end{array}$ & $\begin{array}{c}\text { Hirano, T., \& Murakami, } \\
\text { M. (2020). }\end{array}$ & MEDLINE & Immunity \\
\hline 5 & $\begin{array}{c}\text { Cardiovascular Disease, } \\
\text { Drug Therapy, and } \\
\text { Mortality in Covid-19. }\end{array}$ & $\begin{array}{c}\text { Mehra, M. R., Desai, S. S., } \\
\text { Kuy, S., Henry, T. D., \& } \\
\text { Patel, A. N. (2020). }\end{array}$ & MEDLINE & $\begin{array}{l}\text { New England } \\
\text { Journal of } \\
\text { Medicine }\end{array}$ \\
\hline 6 & $\begin{array}{c}\text { COVID19 Prevention \& } \\
\text { Care; A Cancer Specific } \\
\text { Guideline. }\end{array}$ & $\begin{array}{l}\text { Motlagh, A., Yamrali, M., } \\
\text { Azghandi, S., Azadeh, P., } \\
\text { Vaezi, M., Ashrafi, F., ... \& } \\
\text { Seifi, S. (2020). }\end{array}$ & MEDLINE & $\begin{array}{c}\text { Archives of Iranian } \\
\text { Medicine }\end{array}$ \\
\hline 7 & $\begin{array}{l}\text { COVID-19 Pandemic and } \\
\text { the Crisis of Health } \\
\text { Systems: The Experience } \\
\text { of the Apulia Cancer } \\
\text { Network and of the } \\
\text { Comprehensive Cancer } \\
\text { Center Istituto Tumori } \\
\text { "Giovanni Paolo II" of } \\
\text { Bari. }\end{array}$ & $\begin{array}{l}\text { Silvestris, N., Moschetta, } \\
\text { A., Paradiso, A., \& } \\
\text { Delvino, A. (2020). }\end{array}$ & MEDLINE & $\begin{array}{l}\text { International } \\
\text { Journal of } \\
\text { Environmental } \\
\text { Research and } \\
\text { Public Healt }\end{array}$ \\
\hline 8 & $\begin{array}{c}\text { Pulmonary pathology of } \\
\text { early phase } 2019 \text { novel } \\
\text { coronavirus (COVID-19) } \\
\text { pneumonia in two patients } \\
\text { with lung cancer. }\end{array}$ & $\begin{array}{l}\text { Tian, S., Hu, W., Niu, L., } \\
\text { Liu, H., Xu, H., \& Xiao, S. } \\
\text { Y. (2020). }\end{array}$ & Science Direct & $\begin{array}{c}\text { Journal of Thoracic } \\
\text { Oncology. }\end{array}$ \\
\hline
\end{tabular}


Research, Society and Development, v. 9, n. 7, e628974609, 2020

(CC BY 4.0) | ISSN 2525-3409 | DOI: http://dx.doi.org/10.33448/rsd-v9i7.4609

\begin{tabular}{|c|c|c|c|c|}
\hline 9 & $\begin{array}{l}\text { Management of a colon } \\
\text { cancer patient infected } \\
\text { with corona virus disease } \\
2019 .\end{array}$ & $\begin{array}{c}\text { Ye, Z., Hong, Y., Wu, X., } \\
\text { Hong, D., Zhang, Y., } \\
\text { Dong, X., ... \& Lu, X. } \\
\text { (2020). }\end{array}$ & MEDLINE & $\begin{array}{c}\text { Journal of Zhejiang } \\
\text { University } \\
\text { (Medical Science) }\end{array}$ \\
\hline 10 & $\begin{array}{l}\text { Prostate cancer } \\
\text { radiotherapy } \\
\text { recommendations in } \\
\text { response to COVID-19. }\end{array}$ & $\begin{array}{l}\text { Zaorsky, N. G., James, B. } \\
\text { Y., McBride, S. M., Dess, } \\
\text { R. T., Jackson, W. C., } \\
\text { Mahal, B. A., ... \& Mitin, } \\
\text { T. (2020). }\end{array}$ & Science Direct & $\begin{array}{l}\text { Advances in } \\
\text { radiation oncology. }\end{array}$ \\
\hline 11 & $\begin{array}{l}\text { Clinical Characteristics } \\
\text { and Coping Strategies of } \\
\text { Neoplasms with } 2019 \\
\text { Novel Coronavirus } \\
\text { Infection. }\end{array}$ & $\begin{array}{l}\text { Zhao, N., Shi, J., Zeng, L., } \\
\text { \& Yang, S. (2020). }\end{array}$ & MEDLINE & $\begin{array}{l}\text { Zhongguo fei ai za } \\
\text { zhi= Chinese } \\
\text { journal of lung } \\
\text { cancer. }\end{array}$ \\
\hline
\end{tabular}

Fonte: (Autores, 2020).

O Quadro 2 descreve todos os artigos finais selecionados para o estudo, com autor e ano, tipo de estudo, objetivo e conclusão. 
Quadro 2: Descrição dos artigos por autor e ano, tipo de estudo, objetivo e conclusão.

\begin{tabular}{|c|c|c|c|}
\hline $\mathbf{N}^{\circ}$ & TIPO DE ESTUDO & OBJETIVO & CONCLUSÃO \\
\hline 1 & $\begin{array}{c}\text { Estudo observacional } \\
\text { transversal }\end{array}$ & $\begin{array}{l}\text { Estimar a prevalência de infecção } \\
\text { por vírus respiratórios em } \\
\text { pacientes pediátricos com câncer } \\
\text { e infecção respiratória aguda } \\
\text { (IRA) e / ou febre. }\end{array}$ & $\begin{array}{l}\text { A prevalência de vírus respiratórios } \\
\text { foi relevante no episódio infeccioso, } \\
\text { sem aumento da morbimortalidade. A } \\
\text { co-detecção viral foi frequente em } \\
\text { pacientes com câncer e IRAs. }\end{array}$ \\
\hline 2 & $\begin{array}{l}\text { Estudo observacional } \\
\text { prospectivo com } \\
\text { utilização de } \\
\text { questionário }\end{array}$ & $\begin{array}{l}\text { Coletar informações sobre os } \\
\text { resultados terapêuticos de } \\
\text { prescrições médicas } \\
\text { homeopáticas em pacientes } \\
\text { suspeitos ou confirmados } \\
\text { laboratorialmente com o } \\
\text { diagnóstico de Doença do } \\
\text { Coronavírus (COVID-19) que } \\
\text { demandem espontaneamente } \\
\text { atendimento por médicos } \\
\text { homeopatas. }\end{array}$ & $\begin{array}{c}\text { Alteração do escore global } \\
\text { individualizado de COVID-19, } \\
\text { calculado nas diversas entrevistas } \\
\text { com o paciente, com intervalos } \\
\text { máximos de uma semana, durante um } \\
\text { período de até } 4 \text { semanas após a data } \\
\text { primeira consulta. }\end{array}$ \\
\hline 3 & Estudo informativo & $\begin{array}{c}\text { Descrever e o que se sabe até } \\
\text { agora sobre sua apresentação } \\
\text { clínica. }\end{array}$ & $\begin{array}{l}\text { O vírus responsável pelo COVID-19, } \\
\text { SARS-CoV-2, está na espécie vírus } \\
\text { corona tipo SARS. A } 125 \mathrm{~nm} \text {, é um } \\
\text { pouco maior que os vírus influenza, } \\
\text { SARS e MERS. }\end{array}$ \\
\hline 4 & Revisão da Literatura & $\begin{array}{l}\text { identificam ACE2 como um } \\
\text { receptor SARS-CoV-2, e este } \\
\text { último mostra que seu mecanismo } \\
\text { de entrada depende da serina } \\
\text { protease celular TMPRSS2. }\end{array}$ & $\begin{array}{l}\text { Esses resultados podem explicar a } \\
\text { liberação pró-inflamatória de } \\
\text { citocinas pela via da angiotestina II } \\
\text { associada e um possível alvo } \\
\text { terapêutico pelo eixo da IL-6-STAT3. }\end{array}$ \\
\hline 5 & Estudo Observacional & $\begin{array}{l}\text { Descrever o potencial efeito } \\
\text { prejudicial dos inibidores da } \\
\text { enzima conversora da } \\
\text { angiotensina (ECA) e dos } \\
\text { bloqueadores dos receptores da } \\
\text { angiotensina (BRA) neste } \\
\text { contexto clínico. }\end{array}$ & $\begin{array}{c}\text { Doença cardiovascular subjacente } \\
\text { está associada a um risco aumentado } \\
\text { de morte hospitalar entre pacientes } \\
\text { hospitalizados com Covid-19. }\end{array}$ \\
\hline 6 & Discussão nominal & $\begin{array}{l}\text { Fornecer recomendações e } \\
\text { possíveis ações que devem ser } \\
\text { consideradas pelos pacientes, } \\
\text { seus cuidadores e familiares, } \\
\text { médicos, enfermeiros, gerentes e } \\
\text { funcionários dos centros médicos } \\
\text { envolvidos no diagnóstico e } \\
\text { tratamento do câncer. }\end{array}$ & $\begin{array}{c}\text { Pacientes com câncer requerem } \\
\text { medidas de nível mais alto para } \\
\text { prevenir e tratar doenças infecciosas. } \\
\text { Além disso, os pacientes com câncer } \\
\text { podem sofrer riscos adicionais devido } \\
\text { à restrição de acesso aos serviços } \\
\text { diagnósticos e terapêuticos de rotina } \\
\text { durante essa epidemia. Como a maior } \\
\text { parte da atenção dos sistemas de } \\
\text { saúde é voltada para os pacientes } \\
\text { afetados pelo COVID-19. }\end{array}$ \\
\hline
\end{tabular}




\begin{tabular}{|c|c|c|c|}
\hline 7 & Revisão da Literatura & $\begin{array}{c}\text { Descrever a importância de várias } \\
\text { deficiências evidentes (ou seja, a } \\
\text { ausência planos de gestão de } \\
\text { pandemia, a grave falta de } \\
\text { pessoal e de equipamentos de } \\
\text { proteção). }\end{array}$ & $\begin{array}{l}\text { Demostramos montar uma forte } \\
\text { resposta às necessidades dos } \\
\text { pacientes com câncer durante a } \\
\text { pandemia de COVID-19, } \\
\text { disponibilizando habilidades de } \\
\text { médicos e pesquisadores da região. }\end{array}$ \\
\hline 8 & Relato de caso & $\begin{array}{l}\text { Relatar dois pacientes que foram } \\
\text { submetidos recentemente a } \\
\text { lobectomias pulmonares por } \\
\text { Adenocarcinoma encontrados } \\
\text { retrospectivamente como tendo } \\
\text { COVID-19 no momento da } \\
\text { operação. }\end{array}$ & $\begin{array}{l}\text { Os dois casos fornecem, assim, } \\
\text { importantes oportunidades iniciais } \\
\text { para o estudo da patologia do } \\
\text { COVID-19. Os exames patológicos } \\
\text { revelaram que, além dos tumores, os } \\
\text { pulmões de ambos os pacientes } \\
\text { exibiam edema, exsudato proteico, } \\
\text { hiperplasia reativa focal de } \\
\text { pneumócitos com infiltração celular } \\
\text { inflamatória irregular e células } \\
\text { gigantes multinucleadas. }\end{array}$ \\
\hline 9 & Desfecho clínico & $\begin{array}{c}\text { Explorar a viabilidade do } \\
\text { tratamento cirúrgico para } \\
\text { pacientes com câncer } \\
\text { complicados com a doença do } \\
\text { vírus corona } 2019 \text { (COVID-19). }\end{array}$ & $\begin{array}{l}\text { O caso indica que é possível se } \\
\text { submeter a uma cirurgia radical de } \\
\text { tumor em pacientes com câncer com } \\
\text { COVID-19 após o teste de ácido } \\
\text { nucleico do vírus ficar negativo e } \\
\text { mais estudos são necessários para } \\
\text { confirmar esta conclusão. }\end{array}$ \\
\hline 10 & Revisão sistemática & $\begin{array}{c}\text { Estabelecer recomendações e uma } \\
\text { estrutura para avaliar as decisões } \\
\text { de manejo da radioterapia da } \\
\text { próstata. }\end{array}$ & $\begin{array}{l}\text { Os recursos podem ser reduzidos para } \\
\text { todos os estágios identificados do } \\
\text { câncer de próstata. A estrutura RADS } \\
\text { (visitas remotas e prevenção, } \\
\text { adiamento e encurtamento da } \\
\text { radioterapia) pode ser aplicada a } \\
\text { outros locais de doenças para ajudar } \\
\text { na tomada de decisões em uma } \\
\text { pandemia global. }\end{array}$ \\
\hline 11 & Revisão da Literatura & $\begin{array}{c}\text { Identificação precoce de } \\
\text { pacientes com tumor com a nova } \\
\text { doença de coronavírus } 2019 \\
\text { (COVID-19) e a compreensão de } \\
\text { suas características de } \\
\text { distribuição podem ajudar a } \\
\text { melhorar a taxa de cura dos } \\
\text { pacientes }\end{array}$ & $\begin{array}{l}\text { O SARS-CoV-2 pode transmitir } \\
\text { principalmente por gotículas ou } \\
\text { contato próximo e geralmente é } \\
\text { suscetível à multidão. Pacientes com } \\
\text { tumor apresentam alto risco desse } \\
\text { patógeno devido à sua função } \\
\text { imunológica comprometida. }\end{array}$ \\
\hline
\end{tabular}

Fonte: (Autores, 2020).

O coronavírus é transmitido de pessoa para pessoa por meio de secreções respiratórias e contato, tanto com superfícies como com indivíduos, principalmente através de espirros e 
tosse. Vale a pena ressaltar que uma eventual propagação pode ser possível antes que as pessoas mostrem algum sintoma; porém, essa não é a principal forma de propagação do vírus. O vírus tem um período de latência de 1 a 14 dias, normalmente de 3 a 7 dias. Os sintomas mais presentes são febre, fadiga, tosse seca e falta de ar. Em casos mais graves, pode-se apresentar congestão nasal, náuseas e/ou vômito e diarreia (Motlagh et al., 2020).

A alta taxa de mortalidade da COVID-19 é de 6,7\% em todo o mundo. O desenvolvimento de tratamentos no combate é uma questão urgente e requer parâmetros de qualidade. Hirano \& Murakami (2020) descreveram a Enzima Conversora da Angiotensina 2 (ACE2) como receptores de entrada das células para SARS-CoV-2. O acesso do vírus mediado por receptor depende de uma serina protease, serina protease transmembranar 2 (TMPRSS2). Pode-se notar que os inibidores clinicamente aprovados do TMPRSS2 atuam impedindo a entrada de células por SARS-CoV-2. Como as células alveolares do tipo 2 expressam altamente ACE2 e TMPRSS2 no estado estacionário, essas células dispõe de uma maior facilidade para entrada da SARS-CoV-2 no pulmão, resultado assim em problemas respiratórios.

A Angiotensina II é catalisada pela ACE2 em Angiotensina I, que é conhecida por desempenhar o papel de vasodilatador, anti-inflamatórios e antifibróticos. Foi observado que os inibidores da ECA e os Bloqueadores dos Receptores da Angiotensina (BRA) elevam a expressão da ECA2. Houve relato que os IECA podem contrariar os efeitos anti-inflamatórios da ECA2. Porém, estudos in vitro não apresentam atividade inibitória direta dos inibidores da ECA contra a função da ECA2 (Mehra et al., 2020).

Até o momento, não existe tratamento comprovado na fase inicial de pacientes infectados pelo novo coronavírus. Pode-se notar que abordagens não farmacológicas sejam eficazes, como suporte de fluidos, oxigênio e suporte ventilatório (Fisher \& Heymann, 2020).

Dantas et al. (2020) relata que os diagnósticos clínicos e epidemiológicos na sua maioria são complementados pelo diagnóstico laboratorial por meio do teste de PCR (Polymerase Chain Reaction), conhecido por permitir a amplificação da sequência de RNA do vírus. Além deste, os testes sorológicos com identificação de anticorpos IgM e IgG ao SARS-CoV-2 (testes rápidos) vêm sendo bastante utilizados em profissionais de serviços essenciais durante a pandemia. Outros exames de imagem e laboratoriais poderão ser requisitados, a critério de cada médico e de acordo com as circunstâncias do caso.

A baixa função imune de indivíduos diagnosticados com câncer e a supressão do sistema imunológico sistêmico devido aos tratamentos antitumorais por meio da radioquimioterapia ou tratamento cirúrgico deixa esse paciente mais susceptível a apresentar sintomas severos, caso venha ser infectados por SARS-CoV-2. Em seu estudo, Zhaoet al. (2020) descreveram que, 
aproximadamente, $46,4 \%$ de um total de 138 pacientes com COVID-19 tinham mais de uma doença coexistente. Entre elas, tumores malignos (10/138, 7,2\%), que figuraram como uma das doenças coexistentes mais comuns.

Motlagh et al., (2020) relataram que o Irã foi um dos primeiros países que implementaram uma diretriz nacional direcionada aos pacientes com câncer e COVID-19, ao passo que poucas diretrizes internacionais apresentavam uma série de recomendações de cuidados voltada a estes pacientes. O primeiro grupo de instruções descreveu a respeito das precauções que devem ser tomadas pelos pacientes, enfatizando o fato de que medidas preventivas devem ser tomadas para a maior proteção desta população.

Zhao et al., (2020) descreveram em seu estudo uma pesquisa que analisou os dados epidemiológicos e clínicos de 44.672 casos confirmados de COVID-19. Observou-se que havia 107 pacientes com tumores, representando cerca de 0,5\% do número total de doenças básicas. A taxa bruta de mortalidade foi de cerca de 5,6\%, superior à taxa global de mortalidade bruta $(2,3 \%)$.

Zhang et al., (2020) relataram as características clínicas de 28 pacientes com câncer diagnosticados com COVID-19. O estudo foi realizado em laboratórios de três hospitais designados em Wuhan, China, em 26 de fevereiro de 2020. Cerca de 53,6\% dos pacientes apresentaram eventos graves, 21,4\% foram internados em UTI, 35,7\% dispuseram de complicações com risco de vida e $28,6 \%$ foram a óbito. A taxa de mortalidade de casos chegou a 5,6\% entre os pacientes com câncer, enquanto da população em geral foi de 2,3\%.

Ye et al. (2019) informaram que deve-se formular planos de tratamento razoáveis e monitoramento de medicamentos para melhorar o prognóstico dos pacientes. Silvestris et al. (2020) relataram que os departamentos de Oncologia de radiação devem, portanto, seguir uma série de regulamentos e medidas, para garantir o tratamento oncológico de ponta o maior tempo possível, mantendo sempre a higiene pessoal, do paciente e do dispositivo como medidas importantes em enfermarias, unidades de radioterapia, salas de tratamento de quimioterapia, áreas administrativas, escritórios e todas as outras áreas públicas.

Benites et al. (2020) tiveram como objetivo descrever a prevalência de infecção por vírus respiratórios em pacientes pediátricos com câncer e infecção respiratória aguda (IRA) e/ou febre. Neste estudo, obteu-se a participação de 48 pacientes em tratamento de câncer. 13\% apresentaram sepse bacteriana com IRA.

Tian et al., (2020) relataram o caso de dois pacientes que foram submetidos a lobectomias pulmonares por Adenocarcinoma, ambos diagnosticados com COVID-19 no momento da operação. Com isso, é importante salientar a precaução em laboratórios de 
patologia cirúrgica. Na China, a maior parte das amostras cirúrgicas é recebida fixada em formalina. Porém, para amostras com maior número, o centro de uma amostra pode não estar suficientemente fixo e ainda representar um risco potencial de infecção. Portanto, os equipamentos de proteção individual adequados, como máscaras cirúrgicas ou respiradores N95, por exemplo, devem ser usados o tempo todo na sala grosseira.

O tratamento do câncer na era do COVID-19 dispõe de uma série de riscos e benefícios para pacientes e funcionários. Estudos descrevem que pacientes com câncer têm um risco elevado de infecção e complicações graves do COVID-19. Porém, a Sociedade Americana de Oncologia Clínica fornece recursos para pacientes que recebem terapia sistêmica. A terapia de radiação é distribuída a cerca de 50\% dos pacientes com câncer (Zaorsky et al., 2020).

Zaorsky et al. (2020) relataram que as visitas devem ser realizadas somente por visitas de telessaúde. Porém, ainda que as visitas em vídeo sejam preferidas, essas visitas de telessaúde podem incluir apenas chamadas telefônicas simples, se as visitas em vídeo não forem possíveis devido a limitações de infraestrutura tecnológica em centros selecionados. Isso sugere que os pacientes com tumor são mais propensos à infecção por SARS-CoV-2 e têm um prognóstico pior. A proteção pessoal dos pacientes com câncer deve ser fortalecida, a educação e o treinamento quanto à proteção devem ser realizados e o autogerenciamento dos pacientes deve ser fortalecido.

\section{Considerações Finais}

Conclui-se que os pacientes com tumores apresentam maior risco de COVID-19 que pacientes não-tumorais. Os sintomas restantes são semelhantes aos pacientes não-tumorais: mialgia e fadiga. Contudo, as manifestações de imagem são mais graves e as complicações mais sérias são mais prováveis. Quanto maior a idade, maior o risco de eventos clínicos graves e pior o prognóstico. Quanto mais velhos, mais fácil se desenvolverão em doenças críticas e maior a taxa de mortalidade. Portanto, durante a epidemia, em pacientes idosos com COVID-19 que receberem recentemente radioterapia, quimioterapia ou cirurgia, deve-se ter mais atenção às mudanças na doença e ajustar o plano de tratamento a tempo.

É necessária uma avaliação contínua da triagem para detectar um início precoce dos sintomas típicos de COVID-19 (febre, tosse, dor de garganta, falta de ar e fadiga) em pacientes que já estão recebendo tratamento no Departamento de Oncologia.

Durante essa epidemia, além de uma melhor proteção, os pacientes com câncer precisam de aconselhamento médico on-line, identificação e tratamento adequados de casos críticos. Em 
áreas endêmicas fora de Wuhan, as decisões sobre adiar ou não o tratamento contra o câncer precisam ser tomadas paciente a paciente e de acordo com o risco para o paciente e a situação prevalente, porque os atrasos podem levar à progressão do tumor e, finalmente, a resultados gerais piores.

\section{Referências}

Benites, E. C., Cabrini, D. P., Silva, A. C., Silva, J. C., Catalan, D. T., Berezin, E. N., ... \& Passos, S. D. (2014). Infecções respiratórias virais agudas em pacientes pediátricos com câncer em tratamento quimioterápico. Jornal de Pediatria, 90(4), 370-376.

Dantas, F. (2020). Resultados terapêuticos da homeopatia em pacientes suspeitos ou confirmados de COVID-19 no Brasil: Protocolo para estudo observacional prospectivo. BVSHomeopatia. (pp. 46-46).

Fisher, D., \& Heymann, D. (2020). Q\&A: The novel coronavirus outbreak causing COVID-19. BMC medicine, 18(1), 1-3.

Gil, A. C. (2014). Métodos e técnicas de pesquisa social. 6. ed. São Paulo: Atlas

Hirano, T., \& Murakami, M. (2020). COVID-19: A New Virus, but a Familiar Receptor and Cytokine Release Syndrome. Immunity.

Mehra, M. R., Desai, S. S., Kuy, S., Henry, T. D., \& Patel, A. N. (2020). Cardiovascular Disease, Drug Therapy, and Mortality in Covid-19. New England Journal of Medicine.

Motlagh, A., Yamrali, M., Azghandi, S., Azadeh, P., Vaezi, M., Ashrafi, F., ... \& Seifi, S. (2020). COVID19 Prevention \& Care; A Cancer Specific Guideline. Archives of Iranian Medicine, 23(4), 255-264.

Pereira, A. S., Shitsuka, D. M., Parreira, F. J., \& Shitsuka, R. (2018). Metodologia do trabalho científico. [e-Book]. Santa Maria. Ed. UAB / NTE / UFSM. 
Silvestris, N., Moschetta, A., Paradiso, A., \& Delvino, A. (2020). COVID-19 Pandemic and the Crisis of Health Systems: The Experience of the Apulia Cancer Network and of the Comprehensive Cancer Center Istituto Tumori "Giovanni Paolo II" of Bari. International Journal of Environmental Research and Public Health, 17(8), 2763.

Tian, S., Hu, W., Niu, L., Liu, H., Xu, H., \& Xiao, S. Y. (2020). Pulmonary pathology of early phase 2019 novel coronavirus (COVID-19) pneumonia in two patients with lung cancer. Journal of Thoracic Oncology.

Ye, Z., Hong, Y., Wu, X., Hong, D., Zhang, Y., Dong, X., ... \& Lu, X. (2020). Management of a colon cancer patient infected with corona virus disease 2019. Journal of Zhejiang University (Medical Science), 49(1), 0-0.

Zaorsky, N. G., James, B. Y., McBride, S. M., Dess, R. T., Jackson, W. C., Mahal, B. A., ... \& Mitin, T. (2020). Prostate cancer radiotherapy recommendations in response to COVID-19. Advances in radiation oncology.

Zhao, N., Shi, J., Zeng, L., \& Yang, S. (2020). Clinical Characteristics and Coping Strategies of Neoplasms with 2019 Novel Coronavirus Infection. Zhongguo fei ai za zhi= Chinese journal of lung cancer, 23(4), 261. 
Porcentagem de contribuição de cada autor no manuscrito

Paulo Sérgio da Paz Silva Filho -15\%

Rafael Everton Assunção Ribeiro da Costa -10\%

Monyka Brito Lima dos Santos $-7 \%$

Sonia Pantoja Nascimento Lima $-5 \%$

Leticia de Almeida da Silva $-5 \%$

Ananda Santos Freitas -5\%

Joélio Pereira da Silva $-5 \%$

Laianny Karola Carvalho de Araújo -5\%

Simone Helena Cunha $-5 \%$

Francy Waltilia Cruz Araújo-5\%

Ana Paula Alves da Silva -5\%

Darci Rosane Costa Freitas Alves -5\%

Annarelly Morais Mendes $-5 \%$

Chiara de Aquino Leão -5\%

Cryshna Leticia Kirchesch $-6 \%$

Juliana leal Macedo -7\% 